\title{
W3C Accessibility Guidelines for Mobile Games
}

\author{
Amy Wilson ${ }^{1} \cdot$ Michael Crabb $^{1,2}$ (D)
}

Received: 23 January 2018/Accepted: 22 April 2018/Published online: 2 May 2018

(C) The Author(s) 2018

\begin{abstract}
In the past decade, video games have become one of the fastest growing forms or entertainment around the world. In particular, mobile gaming has continued to evolve, becoming increasingly popular for billions of people worldwide. An ongoing issue within this subject is the accessibility issues users' face when playing these types of games. The purpose of this work is to investigate how well current $\mathrm{W} 3 \mathrm{C}$ accessibility guidelines support mobile game development. This paper introduces a study that was carried out to determine the current knowledge that participants have of these guidelines, along with the opinions on how important these accessibility guidelines are when creating accessible mobile game content.
\end{abstract}

Keywords Accessibility · Mobile gaming · Interview · Guidelines

\section{Introduction}

In recent years, there has been a growing amount of interest in mobile gaming applications. Figures from The Association for UK Interactive Entertainment (UKIE) state that 21.8 billion mobile game applications were downloaded from

All editorial decisions for this article, including selection of reviewers and the final decision, were made by guest editor Dr. Michael Heron.

Michael Crabb

michaelcrabb@acm.org

Amy Wilson

a.j.wilson3@rgu.ac.uk

1 Robert Gordon University, Aberdeen, Scotland, UK

2 University of Dundee, Dundee, Scotland, UK 
Apple's App Store and Google Play in Q1 of 2017-2018, a 15.3\% increase from the findings from Q1 of 2016-2017 (UKIE 2018). As this category of games evolve, there is a growing demand for these games to achieve the maximum level of accessibility for users with disabilities. This will ultimately allow all users to fully engage with the mobile gaming experience.

Mobile gaming is one of the most popular forms of gaming. Billions of people around the world play mobile games on a regular basis and view this activity as an effortless and uncomplicated means of passing time or as a distraction from hectic schedules. $56 \%$ of individuals play mobile games whilst they are in situations where they are waiting (Dobson 2006). This presents the importance of manufacturing these games to become accessible to users in a range of different environments, allowing users to have an engaging experience with these types of games.

One method that can be used to assist in creating accessible games is through the consultation of accessibility guidelines. This has had varying success in fields such as web accessibility (Carter and Fourney 2007), Computer Science education (Rosmaita et al. 2006), and computer games (Ellis et al. 2012). Of particular interest is the World Wide Web Consortiums (W3C) Web Content Accessibility Guidelines (WCAG) which is currently in its second iteration (W3C 2017b). WCAG has been used previously to assist in the creation of accessible education games (Cano et al. 2017) and also in the teaching of games accessibility (Westin and Dupire 2016).

In light of current W3C work examining future iterations of accessibility guidelines (W3C 2017a), this paper attempts to answer the following question:

\subsection{How Well Do W3C Accessibility Guidelines Support Recreational Activities, Such As Games?}

This paper discusses findings from interview sessions on the topic of WCAG usage in creating accessible mobile games. These interview sessions were carried out to gain an insight into the current awareness users have in regard to accessibility guidelines that should be used when designing mobile games. The data gathered from these sessions will be discussed throughout this paper, presenting the most highly recognized guidelines and how well these support mobile games. It will also discuss some of the additional requirements and complications involved for developers when adhering to accessibility guidelines.

\section{Related Work}

The past decade has seen the rapid development of mobile technology in many different social organizations. It is estimated that $75 \%$ of adults own a mobile phone and most feel it would be difficult to give this up (Horrigan 2009). One of the most popular aspects of mobile phones is the apps and games that can be downloaded through the simple touch of a button. Approximately 46 billion mobile applications were downloaded in 2012 between android and iOS devices with this expected to grow by $35.9 \%$ each year (Liu et al. 2015). Recent figures also suggest that over 180 
billion applications have been downloaded from the Apple App Store alone from 2008 to 2017 (Statista 2017).

One of the main obstacles included in the use of mobile applications is the low prevalence of accessible content that is available. The World Wide Web Consortium (W3C) has recently completed a working draft version of the 2.1 version of its Web Content Accessibility Guidelines (WCAG) to make accessible content for users with disabilities (W3C 2017b). Issues exist within current W3C accessibility guidelines when examining their usage with mobile technology and it has been argued that changes are needed in order to improve overall mobile accessibility (Clegg-Vinell, Bailey and Gkatzidou 2014). This view is supported by Cooper (2016) who states that issues specific to mobile devices require further addressing. Compounding this, very little attention has been paid to the role of these guidelines in mobile phone games.

A number of techniques have previously been developed to enhance the accessibility of current mobile phone hardware and software to provide a more accessible and enjoyable experience for users with disabilities. A large majority of these are focused mainly on creating accessible games for users who are blind or have low vision. "VBGhost" is an example of one of these systems. It is an educational smartphone game which integrates the use of braille for children who are blind or have low vision (Milne et al. 2013). Similarly, a system was created for blind and low vision users in which the user interacts with the game through audio cues, rather than visual cues (Kim and Ricaurte 2011). Although the two previously mentioned games focused on blind and low vision users, a game was created in which both sighted and non-sighted gamers could play. By integrating fully accessible menus and a number of optional play levels it allows all users to share the same gaming experience (Glinert and Wyse 2007). There is little published data on the accessibility of mobile games for other common disabilities, however a networked game was created to support the social connection of youth with cerebral palsy (Hernandez et al. 2014).

\section{Methodology}

Previous literature has shown that it is possible to use the $\mathrm{W} 3 \mathrm{C}$ Web Content Accessibility Guidelines (WCAG) when examining the accessibility of mobile content. However, it is unknown to what level current WCAG guidance can assist developers in the creation of accessible mobile games. The aim of this work is to examine the appropriateness of current W3C Web Content Accessibility Guidelines in supporting mobile games and to understand developers' opinions in what additions need to be made in order to increase understanding.

\subsection{Participants}

A total of 6 participants ( 3 male, 3 female) aged between 17 and 23 participated were recruited for this work. Participants were recruited through email and invited to take part in an interview session. Participants were all students in either Computer 
Science or Digital Media based undergraduate university courses and had completed a small amount of accessibility work as part of their relevant degree program. The inclusion of participants from a number of different (but related) degree programmes and from different university stages assists in promoting diversity in thinking that is required in this type of work.

All participants were experienced in software development and used mobile phones daily. Participants also had experience in the design of web content and had some knowledge of the accessibility features that should be included throughout the development of web applications and content. Participants were informed during recruitment that this work would examine the accessibility of mobile games, but were not told the overall procedure of what the work would entail or to what degree their own understanding of accessible design practice would be discussed.

Whist the number of participants in this work is relatively low, as this work is qualitative in nature, the number of participants is deemed acceptable (Kvale 2007b). Additionally, and in an attempt to combat this, all analysis took the number of participants into consideration. Research themes were only identified if they were discussed by a number of participants. Thoughts and opinions of individual participants that were not echoed by others, although potentially relevant, were not included.

\subsection{Design and Procedure}

This work uses one-to-one semi-structured interviews as a method of eliciting information from participants about their experiences and thoughts in using WCAG to design accessible products. Interviews are well suited for data gathering in both retrospective and longitudinal studies (Flick 2007a) with conceptual and factual type interviews (Kvale 2007a) lending themselves well to this type of inquiry.

Each interview was recorded to allow these to be transcribed, ensuring that all relevant information could be recorded during the discussion stage of the interview. Along with this, notes were taken throughout the interviews, noting down any relevant information that may be missed in the recordings of the interviews. This ensured that all data could be recorded from each of the interviews to be referred to at a later stage.

In line with guidance from Flick (2007b), interviews were carried out in a quiet space and were free from distractions throughout.

\subsubsection{Interview Procedure}

Before starting interviews, participants were asked to sign a consent form, required as part of institutional ethical approval. Participants agreed to be audio recorded throughout the interview and were informed that their participation and answers throughout the interview were voluntary. Participants were also informed that the answers they gave throughout the interview would be kept anonymous and would only be used for the purpose of the study. The interviews consisted of a number of tasks which were carried out over a period of $1 \mathrm{~h}$. 
The purpose of the first interview task was to gain an insight into the current knowledge each participant had of existing accessibility guidelines. Participants were asked to note down any accessibility guidelines they were already aware of or believed to be relevant when designing accessible mobile phone games. Participants were given a block of post-it notes on which they were asked to note down accessibility guidelines they were aware of, writing each guideline on a separate post-it note. Participants were given 3 min to note these down. Once this task had been completed, participants were asked to discuss their responses out loud and to explain why they felt these accessibility guidelines were relevant.

The second task participants were asked to complete was to order the accessibility guidelines they had noted down into order of importance. Participants were given 2 min to complete this task and were then asked to discuss their reasoning for ordering the accessibility guidelines in a particular order. The purpose of this was to observe which of the guidelines each participant felt were the most important and relevant when designing accessible web content.

Finally, participants were shown a list of the current $\mathrm{W} 3 \mathrm{C}$ guidelines that exist for designing accessible web content for mobile devices. These guidelines focused on the accessibility of mobile phones in general, including the user interface and browser guidelines developers should adhere to. Participants were given a further 3 min to read through this. The list of guidelines was then discussed with each participant, discussing their opinions of the most important guidelines when designing for mobile games as a specific topic along with their reasoning for ordering these in a particular way.

After showing the participants the list of current W3C accessibility guidelines, they were then asked if they felt there should be any guidelines added or improved on in this list, to allow these guidelines to support more accessibility within mobile games. The interview was then concluded by asking the participants how well they felt the current $\mathrm{W} 3 \mathrm{C}$ accessibility guidelines supported mobile games.

\subsection{Analysis}

The purpose of analysis is to provide a comparison between each of the participants opinions, allowing for recurring themes to be made apparent on the participants opinions of the current W3C accessibility guidelines. Each interview was transcribed and then sorted based on the previously described interview tasks. Transcripts for each task were examined and an inductive-nomothetic approach (Gibbs 2007) was taken. From this, comparative analysis (Rapley and Flick 2007) was conducted in order to examine the connections between participants and to also look for instances when contrasting opinions were present.

\section{Results and Discussion}

This study set out with the aim of finding out how well W3C accessibility guidelines support the development of mobile games. This was evaluated by researching how well-known the current accessibility guidelines are, as well as how important 
participants feel these are, the guidelines these participants would add or improve on and ultimately, how well they feel these guidelines support the development of mobile games.

The data collected from the interviews consists of five parts:

- The current awareness of accessibility guidelines when designing for mobile games;

- The importance of the accessibility guidelines known by participants;

- The importance of existing W3C accessibility guidelines;

- Recommendations for W3C accessibility guidelines in the future, and;

- The overall success of existing W3C accessibility guidelines.

\subsection{Current Awareness}

The purpose of the first task was to gain an insight into the current awareness participants have of existing accessibility guidelines as well as their opinions on the importance of these guidelines. A number of accessibility guidelines were identified by the participants, however a recurrent theme in the interviews was the accessibility guidelines relating to visual impairments. Participants did not mention the Perceivable, Operable, Understandable, Readable (POUR) principles that exist within WCAG and a large amount of comments focused on the visual aspects of accessibility. These views surfaced mainly in relation to users with low vision, as P5 stated:

Because I'm visually impaired, text is bad if you don't have good contrast to the background because the phone is smaller than a computer screen, so you can't see the text as big.

Similarly, P2 stated:

If you've got issues with reading you can make text bigger if there is text on the screen.

Another accessibility guideline identified was guidelines relating to Colour Vision Deficiency (CVD). P1, P2, P3 and P4 all mentioned guidelines which aid users with CVD. As one interviewee said:

Games where you have colourful bubbles and you have to select the ones that are one colour. Often I see those have settings where you can make those not colours but forms like triangles so people who are colour blind can select shapes instead of colours.

A small number of those interviewed also suggested that seizure guidance was an important accessibility guideline (P1 and P5). P1 stated:

Seizures are dangerous and so it is important to avoid these.

This view was echoed by P5 who also found this to be of importance when designing accessible mobile games: 
Seizures can affect anyone at any time so flashing images can catch people out.

A minority of respondents noted accessibility guidelines relating to cognitive disabilities. P2 and P4 mentioned accessibility guidelines that could aid users with cognitive disabilities with P2 stating:

Text to speech translation would be important for people with issues reading so if you've got dyslexia you might not be able to read the text as easily

Whilst participants had very clear views on visual accessibility challenges, it became clear in all interviews that there was a limited amount of knowledge participants on other aspects of accessibility guidelines. The results gathered from this section showed that the overall current knowledge of WCAG is minimal for this set of participants.

This view is supported by Shneiderman and Hochheiser (2001), stating that there are gaps in user knowledge in relation to designing programs which are universally usable and accessible. Further development should be undertaken to ensure that the overall awareness of all aspects of accessibility guidelines should be focused on. It is important to ensure that all developers are aware of these and can refer to these when designing accessible media.

\subsection{Importance of Known Accessibility Guidelines}

After learning the participants initial awareness of accessibility guidelines, participants were asked to order the guidelines they had noted into order of importance. Participants were asked:

Which of these guidelines do you feel are the most important when designing accessible mobile games?

Opinions differed on the importance of accessibility guidelines relating to visual impairments. Of the 5 participants who noted guidelines relating to visual impairments, only 3 of these ordered these guidelines as being the most important when designing accessible mobile games. All participants stated these guidelines as being important, however 2 participants stated that other guidelines may be of higher importance when designing accessible mobile game content. An example of this is P5, ordering seizure guidance as the most important:

Seizures can cause bad health and is a health risk.

The importance of accessibility guidelines relating to CVD were also differed as P4 stated this as the highest importance, whilst P2 and P3 stated this as the lowest importance. P3 stated:

People who have colour blindness [CVD] can still see the game, so they are not missing out on as much as people with other disabilities.

A common view amongst interviewees was that seizure guidance was an important accessibility guideline and should be included throughout the design of mobile 
games. P1 ordered this as being the second most important guideline whilst P5 ordered this as being the most important guideline. P1 stated:

You don't want to harm someone's health by designing something harmful.

Of the two participants that noted accessibility guidelines relating to cognitive issues, both felt that these guidelines were not of the highest importance when designing accessible mobile games. Both P2 and P4 ordered these specific guidelines as being the second most important when designing these types of games.

Throughout the interview sessions, only one participant noted accessibility guidelines relating to hearing impairments in the current awareness task of the interviews. When ordering the guidelines in order of importance this participant ordered guidelines relating to hearing disabilities as being the most important.

Taken together, these results suggest the opinions each participant has of the importance of accessibility guidelines when designing accessible mobile games, allowing insight into how well the $\mathrm{W} 3 \mathrm{C}$ accessibility guidelines support these types of games.

The findings from this section of the interviews highlighted the opinions from participants on the importance of the accessibility guidelines they had noted in the previous task. Opinions differed as to which of the noted guidelines were of highest importance when designing mobile gaming applications. A common theme was the importance of visual impairment accessibility guidelines. P1, P2, P4, P5 and P6 all stated that accessibility guidelines which aid visual impairments were of highest importance.

Similar to the results that we found, Clegg-Vinell et al. (2014) discuss users in their work frequently reported issues relating to contrast. A possible explanation for this common theme could be due to the awareness of current accessibility issues and guidelines. Whilst the majority of users felt that guidelines aiding visual impairments was of highest importance, it can be assumed that this particular issue, along with the guidelines to aid this issue is the most well-known. The most striking result to emerge from the data was that only one participant noted accessibility guidelines which aid hearing impairments. When ordering, this participant ordered these highest in importance, whilst only two of the participants mentioned the importance of seizure accessibility guidelines. It can therefore be assumed that these guidelines are less known that those relating to visual impairments, highlighting a need for other, less-known guidelines to become more apparent to developers when designing accessible mobile game content.

\subsection{Importance of Current Accessibility Guidelines}

The next part of the interviews asked participants to pick out what they felt to be the most important aspects of WCAG relating to mobile games. Participants were given a list of W3C accessibility guidelines for creating accessible mobile content.

The majority of participants stated that guidelines included in both the perceivable and operable sections were of highest importance when designing accessible mobile games. A common view amongst interviewees was the importance of button size and layout within these types of games. Some felt that 
accessible buttons were of high importance, while others considered that touch target size were also of high importance. P1 and P6 stated that easily accessible buttons were an important guideline with $\mathrm{P} 1$ stating:

Some people struggle to reach certain buttons, so buttons should be suitable to everyone.

The importance of accessible buttons was echoed by P4 who stated that touch target size was of high importance:

People with disabilities should be able to touch buttons with ease.

Another recurrent theme in the interviews was the importance of gesture accessibility, including touchscreen gestures and device manipulation gestures. P3, P4 and P6 all stated that these guidelines were of high importance. P4 stated that there should be alternatives for device manipulation gestures:

Devices that are in fixed positions are unable to be tilted so having other features are useful for example buttons to simulate tilting.

This view was echoed by P3 who stated:

Users who can't do these gestures should be provided with alternatives.

Surprisingly, only a minority of the respondents stated that accessibility guidelines relating to visual impairments were of high importance. P2 and P5 stated that the zoom/magnification guideline was of high importance as it can aid users with low vision by making the text bigger onscreen.

Participants indicated that guidelines included in the perceivable and operable sections of the W3C accessibility guidelines were of highest importance when designing accessible mobile games. Button size and layout was a recurring theme amongst participants. Similarly, the importance of gesture accessibility was a popular response from participants. Each of these accessibility guidelines are put in place to mainly aid those who have motor impairments. The importance of creating accessible web content for these types of users is echoed by Malu and Findlater (2015), creating a head-mounted display for users with upper body motor impairments. Similarly, Hornof (2009) states how to design for children with motor impairments, expressing the difficulty of designing for these types of disabilities.

One interesting finding was that only two of the participants interviewed mentioned accessibility guidelines relating to visual impairments despite this category being very prominent during the first part of the study. These findings may help to understand which of the current accessibility guidelines should be improved on for future developments. From the data collected in this section of the interviews, it can be assumed that participants feel there should be more development around accessibility guidelines which aid motor impairments and should be more frequently integrated into mobile gaming applications. 


\subsection{Future Accessibility Guidelines Recommendations}

Participants were then asked to discuss recommendations for accessibility guidelines, stating any guidelines they feel had been missed out or should be improved on for the future. The majority of those who responded to this felt that there should be more consideration for CVD throughout the $\mathrm{W} 3 \mathrm{C}$ accessibility guidelines. P1, P2 and P3 all stated that colour schemes should be considered and should have an option to be made changeable to aid users who have CVD. P1 stated that colour should be considered for both CVD as well as autism, possibly referring to high levels of CVD present within individuals with autism spectrum disorders (Zachi et al. 2017).

Another recurring theme from the responses for this question was the readability aspect for people with visual impairments and/or dyslexia. P1 stated that there should be an option integrated to read out rules and notifications to users, whilst P2 stated that the text to speech translation should be integrated into more games:

Text to speech translation would be helpful. I mean some games do read things out but some don't. Text being read out would be helpful to people with dyslexia.

One interviewee reported that seizure guidance should be improved on when designing mobile games. P5 commented:

More games should make sure there are no flashing images for people with epilepsy.

Surprisingly, only one participant mentioned an accessibility guideline from the understandable section of the W3C accessibility guidelines. P4 said:

Not many games incorporate orientation changes. Some people can only hold phone in one way.

These results suggest that the majority of participants agreed that there should be accessibility guidelines added or improved on to allow for more accessible mobile game applications.

The responses gathered from this section of the interviews followed two recurring themes. The majority of participants interviewed stated that the accessibility guidelines have more focus on CVD aids as well as seizure guidance to aid those with epilepsy. These findings suggest that there are not enough accessibility guidelines relating to each of these disabilities. This view is supported by P6, whilst in their interview session they stated:

I would probably never of even considered these to be a big deal until we had

a look through the extensions on google chrome.

From this it can be assumed that aids for these types of disabilities can be added as extensions on web browsers, however there is a lack of accessibility for these integrated in mobile gaming applications. 


\subsection{Overall Support for Mobile Games}

In the final part of the interview, participants were asked how well current $\mathrm{W} 3 \mathrm{C}$ accessibility guidelines support the development of mobile games.

A recurrent theme in the interviews was a sense amongst interviewees that the current $\mathrm{W} 3 \mathrm{C}$ accessibility guidelines exceptionally support mobile games. P1, P2, P3, P4 and P6 were all in agreement that the $\mathrm{W} 3 \mathrm{C}$ accessibility guidelines support mobile game development as much as possible. P1 commented that the only thing that seemed to be missing was seizure guidance, whilst P3 commented that there should be some more incorporation of CVD aids. P2 commented on the fact that a lot of games incorporate these guidelines, whilst some others do not. In agreement with this, P4 stated:

A lot of the operable guidelines are already incorporated in most of the games. When I read them, images come to my mind of games I have played that incorporate them.

One interviewee argued that these $\mathrm{W} 3 \mathrm{C}$ accessibility guidelines do not support the development of mobile games, but this focused more on overall layout and understanding of the guidelines themselves. P5 stated:

The $\mathrm{W} 3 \mathrm{C}$ accessibility guidelines are hard to find when searching for them.

It's like an encyclopaedia with no index.

The overall response to this section of the interview is rather encouraging, as the majority of participants indicated that the current $\mathrm{W} 3 \mathrm{C}$ accessibility guidelines supported mobile games exceptionally well, gaining the overall impression that these guidelines support these types of games as much as possible.

One participant stated that some games incorporate these accessibility guidelines whilst others do not. This view is supported by Jaramillo-Alcazar and Lujan-Mora (2017) who state that accessibility for users with visual impairments should be considered more by mobile games developers.

However, one of the participants disagreed with this and stated that the current W3C accessibility guidelines did not support the development of mobile gaming applications. The findings from this suggest that overall, the W3C accessibility guidelines support the development of mobile games relatively well, whilst more games should focus on incorporating these guidelines to improve the overall accessibility of mobile game content.

\section{Conclusion}

This study set out to gain an insight into how well the current $\mathrm{W} 3 \mathrm{C}$ accessibility guidelines support recreational activities such as mobile gaming applications. Results from the interview highlight issues relating to these guidelines. The need for current accessibility guidelines to be made more available and apparent to mobile game developers was highlighted. Along with this, it was highlighted that accessibility guidelines which aid other impairments should be made more apparent 
and incorporated more frequently throughout the development of mobile gaming applications.

Awareness of the full spectrum of accessibility areas covered by WCAG needs to be increased. Participants in this work were very quick to discuss accessibility issues related to vision but had little knowledge of any other areas. It is important that future accessibility guidelines find methods to demonstrate the full spectrum of accessibility areas that users can face and use this as a method to assist developers in understanding how to create more accessible products.

The W3C WCAG can be used to assist with accessibility of mobile games in its current state but caution must be applied. In general, it seems that the W3C accessibility guidelines support the development of mobile games relatively well, however there are a number of improvements required to ensure these guidelines cover all accessibility issues users may encounter when accessing mobile games. Areas such as movement-based applications and AR games require additional guidelines in order to assist in overcoming accessibility challenges.

It is clear that there is benefit in the use of accessibility guidelines such as WCAG in the creation of accessible games and other services. However, additional work should be carried out in order to better understand the accessibility challenges that can affect the creation of accessible mobile games. Whilst the current Web Accessibility Guidelines can be used as a temporary solution, additional work is required if they are to be used successfully outside of the web domain and into recreational areas such as mobile games.

\section{Compliance with Ethical Standards}

Conflict of interest On behalf of all authors, the corresponding author states that there is no conflict of interest in this work.

Open Access This article is distributed under the terms of the Creative Commons Attribution 4.0 International License (http://creativecommons.org/licenses/by/4.0/), which permits unrestricted use, distribution, and reproduction in any medium, provided you give appropriate credit to the original author(s) and the source, provide a link to the Creative Commons license, and indicate if changes were made.

\section{References}

Cano, A. R., Fernández-Manjón, B, \& García-Tejedor, Á. J. (2017). GLAID: Designing a game learning analytics model to analyze the learning process in users with intellectual disabilities. In Lecture Notes of the Institute for Computer Sciences, Social-Informatics and Telecommunications Engineering, LNICST, pp. 45-52. https://doi.org/10.1007/978-3-319-51055-2_7.

Carter, J. A., \& Fourney, D. W. (2007). Techniques to assist in developing accessibility engineers. In Proceedings of the 9th international ACM SIGACCESS conference on computers and accessibility Assets 07 (pp. 123-130). https://doi.org/10.1145/1296843.1296865.

Clegg-Vinell, R., Bailey, C., \& Gkatzidou, V. (2014). Investigating the appropriateness and relevance of mobile web accessibility guidelines. https://doi.org/10.1145/2596695.2596717.

Cooper, M. (2016). Web accessibility guidelines for the 2020 s.

Dobson, J. (2006). Gamasutra-survey: Global mobile gaming, purchasing habits analyzed.

Ellis, B., Ford-Williams, G., Graham, L., Grammenos, D., Hamilton, I., Headstrong Games, Lee, E., Manion, J., \& Westin, T. (2012). Game accessibility guidelines. Available at http:// gameaccessibilityguidelines.com/full-list. Accessed 28 August 2017. 
Flick, U. (2007a). Designing qualitative research. The Sage Qualitative Research Kit. https://doi.org/10. $4135 / 9781849208826$.

Flick, U. (2007b). Managing quality in qualitative research. The Sage Qualitative Research Kit. https:// doi.org/10.4135/9781849209441.n8.

Gibbs, G. (2007). Analyzing Qualitative Data, SAGE Qualitative Research Kit.

Glinert, E., \& Wyse, L. (2007). AudiOdyssey: An accessible video game for both sighted and non-sighted gamers, pp. 251-252.

Hernandez, H. A., Ketcheson, M., Schneider, A., Ye, Z., Fehlings, D., Switzer, L., Wright, V., Bursick, S. K., Richards, C., \& Graham, N. (2014). Design and evaluation of a networked game to support social connection of youth with cerebral palsy, pp. 161-168.

Hornof, A. J. (2009). Designing with children with severe motor impairments. Systems for Children, pp. $2177-2180$.

Horrigan, J. B. (2009). The Mobile Difference.

Jaramillo-Alcazar, A., \& Lujan-Mora, S. (2017). Mobile serious games: An accessibility assessment for people with visual impairments. Alicante.

Kim, J., \& Ricaurte, J. (2011). TapBeats: Accessible and mobile casual gaming, pp. 285-286.

Kvale, S. (2007a). Doing Interviews. https://doi.org/10.4135/9781849208963.

Kvale, S. (2007b). Introduction to interview research. Sage Online Research Methods, pp. 2-10. https:// doi.org/10.4135/9781849208963.

Liu, W., Zhang, G., Chen, J., Zou, Y., \& Ding, W. (2015). A measurement-based study on application popularity in Android and iOS App Stores, pp. 13-18. https://doi.org/10.1145/2757384.2757392.

Malu, M., \& Findlater, L. (2015). Personalized, wearable control of a head-mounted display for users with upper body motor impairments. HMDs \& Wearables to Overcome Disabilities, pp. 221-230. https://doi.org/10.1145/2702123.2702188.

Milne, L. R., Bennet, C. L., \& Ladner, R. E. (2013). VBGhost: A Braille-based educational smartphone game for children. Seattle. https://doi.org/10.1145/2513383.2513396.

Rapley, T., \& Flick, U. (2007). Doing conversation, discourse and document analysis. In Doing conversation, discourse and document analysis. https://doi.org/10.4135/9781849208901.

Rosmaita, B. J., Deibel, K., Cohen, R. F., \& Egan, M. A. L. (2006). Accessibility and computer science education. ACM SIGCSE Bulletin, 38(1), 63. https://doi.org/10.1145/1124706.1121363.

Shneiderman, B., \& Hochheiser, H. (2001). Universal Usability as a Stimulus to Advanced Interface Design.

Statista. (2017). Apple Store Download Statistics. Available at https://www.statista.com/statistics/263794/ number-of-downloads-from-the-apple-app-store/. Accessed 1 January 2017.

UKIE. (2018). UK Video Games Fact Sheet. https://doi.org/10.1017/cbo9781107415324.004.

Westin, T., \& Dupire, J. (2016). Evaluation and redesign of a curriculum framework for education about game accessibility. In Lecture Notes in Computer Science (including subseries Lecture Notes in Artificial Intelligence and Lecture Notes in Bioinformatics) (pp. 217-222). Cham: Springer. https:// doi.org/10.1007/978-3-319-46100-7_20.

W3C. (2017a). Silver Task Force. Available at https://www.w3.org/WAI/GL/task-forces/silver/. Accessed 19 January 2018.

W3C. (2017b). Web content accessibility guidelines (WCAG) 2.1. Available at https://www.w3.org/TR/ WCAG21/. Accessed 10 January 2018.

Zachi, E. C., Costa, T. L., Barboni, M. T. S., Costa, M. F., Bonci, D. M. O., \& Ventura, D. F. (2017). Color vision losses in autism spectrum disorders. Frontiers in Psychology. Frontiers Media, SA, 8, p. 1127. https://doi.org/10.3389/fpsyg.2017.01127. 